\title{
Entretien avec Paul Golub, metteur en scène
}

\section{Paul Golub et Isabelle Schwartz-Gastine}

\section{OpenEdition \\ Journals}

\section{Édition électronique}

URL : http://journals.openedition.org/shakespeare/1350

DOI : 10.4000/shakespeare.1350

ISSN : 2271-6424

Éditeur

Société Française Shakespeare

Édition imprimée

Date de publication : 1 novembre 2002

Pagination : 223-235

Référence électronique

Paul Golub et Isabelle Schwartz-Gastine, «Entretien avec Paul Golub, metteur en scène », Actes des congrès de la Société française Shakespeare [En ligne], 19 | 2002, mis en ligne le 01 novembre 2007, consulté le 21 avril 2019. URL : http://journals.openedition.org/shakespeare/1350 ; DOI : 10.4000/ shakespeare. 1350 


\section{S H A K E S P E A R E \\ \& L E M O Y E N - Â G E}

Société Française Shakespeare

Actes du Congrès de 2001

* * *

Textes réunis et présentés par

Patricia DORVAL

publiés sous la direction de Jean-Marie MAGUIN 


\section{ENTRETIEN A E C PAUL GOLUB, METTEUR EN S CEN E}

\section{Présentation}

D'origine américaine, mais parfaitement bilingue puisqu'il a suivi sa scolarité dans un lycée français, Paul Golub est venu en Europe en 1985 étudier le théâtre contemporain grâce à une bourse accordée par le prix Watson qui récompense un jeune acteur prometteur. Après avoir voyagé en Italie, il a suivi un stage au Théâtre du Soleil, où il est resté trois ans. Il a tenu divers petits rôles dans les deux grandes épopées écrites par Hélène Cixous, L'Histoire terrible, mais inachevée de Norodom Sianouk, roi du Cambodge (1985) et L'Indiade ou l'Inde de leurs rêves (1987). En plus de son expérience de comédien, il y a accumulé des connaissances techniques de mise en scène et de direction d'acteurs. Il a également retenu le sens primordial du corporel, l'importance de l'entraînement physique pour une maîtrise toujours plus exigeante des possibilités de chacun, l'implication totale du corps, du mental, de la voix. Il se plaît à mentionner également comme principe théâtral le plaisir du jeu partagé dans une création qui laisse une large place à la créativité de tous les membres participants.

En 1990, il crée sa propre compagnie, La Compagnie du Volcan Bleu, avec une structure administrative permanente et une participation ponctuelle mais fidélisée d'acteurs rencontrés au Théâtre du Soleil, dont Simon Abkarian et Clémentine Yelnik, qui ont donc la même conception de l'acte théâtral, ainsi que la costumière qui imprime le même goût pour 
les étoffes flottantes et les couleurs éclatantes. À partir de 1997, Le Volcan Bleu reçoit des subventions de la DRAC du Limousin selon une convention triennale.

Il se propose de faire connaître des auteurs contemporains, comme Caryl Churchill, dramaturge anglaise dont les pièces, Fen, aimablement traduite pour l'occasion par Claude Duneton, et Septième Ciel, dans une traduction de Jean-Pierre Carasco, ont été toutes deux créées au Théâtre du Chaudron à la Cartoucherie de Vincennes, en 1990 et en 1992, avant d'effectuer des tournées en province. C'est avec une pièce de l'auteur africain Koulsy Lamko, Tout Bas... Si Bas, interprétée principalement par des acteurs africains de diverses nationalités qu'il a ouvert le Festival International des Francophonies de Limoges en 1998, avant d'aller à Villeneuve-les-Avignon et Alençon. En 1994, il a exploré le devoir de mémoire dans une adaptation du récit de Vercors, Les Armes de la nuit, effectuée et interprétée par Jean-François Dusigne, d'abord au Théâtre du Soleil dont il est issu, puis en tournée. Cette mise en scène a été remarquée par sa sobriété et son tact qui donnait dignité et vérité au récit de ce chef de réseau de la résistance, qui au retour de déportation, après tortures et interrogatoires, ne pouvait retrouver sa place parmi les siens.

Inspiré par la commedia dell'arte d'après des farces de Molière, Il Circo Popolare Poquelino a été créé au Festival de la Luzège en Corrèze en 1993, festival auquel Paul Golub va également participer en 1995 avec sa première pièce de Shakespeare, Le Songe d'une nuit d'été, dans la traduction de Stuart Seide. Cette mise en scène y remporte un franc succès : «Shakespeare débridé», «version jubilatoire», «un songe éveillé», «un théâtre enchanteur qui est également une véritable fête», et après une tournée en France, elle va être interprétée à Paris en 1997, au Théâtre du Lierre, en alternance avec une tragédie cette fois, Macbeth, (traductions de Jean-Michel Déprats). Ce sont les mêmes acteurs qui vont intervenir dans les deux productions, si bien que d'un soir à l'autre, Simon Abkarian passe de Bottom et Égée à Macbeth, David Ayala de Puck à Malcolm, Clémentine Yelnik de Philostrate, la Fée et Snout à la première sorcière et au Portier et Catherine Schaub de Titania et Hippolyta à Lady Macbeth. Le Songe a reçu le prix du meilleur spectacle de l'année 1997 décerné par l'Association des Étudiants de la Sorbonne, ainsi que le prix du meilleur acteur dans un premier rôle et celui de la meilleure actrice dans un second rôle.

Ce spectacle comportait treize comédiens qui se partageaient les rôles de façon maintenant traditionnelle entre le royaume des Fées et le duché 
d'Athènes. Mais la particularité concerne les six artisans qui exprimaient, dans leur ardeur de comédiens amateurs, le principe du plaisir théâtral partagé, principe déjà énoncé cher à Paul Golub. C'est l'idée de la fête qui les guide, et le plaisir de se retrouver ensemble pour jouer, même si, selon le fil conducteur qui a alimenté son jeu, Clémentine Yelnik m'a dit qu' «ils vivent dans le risque de la mort s'ils font pleurer les dames, ils sont menacés par la tyrannie d'Athènes tout cela pour leur passion du théâtre». Pour les représenter, Paul Golub s'est inspiré de figures clownesques traditionnelles, comme l'Auguste, c'est pourquoi ils étaient très maquillés, le visage blanc avec des touches vives sur les joues et sur le nez, contrastes que les comédiens ont atténué au fur et à mesure des représentations. Il s'est aussi tourné vers le cinéma et les héros comiques de films muets, de Laurel et Hardy à Charlot, ce qui explique les costumes noirs sur chemise blanche. Le folklore juif d'Europe Centrale affleure également, dans la tendance rétro que les comédiens évoquent et surtout dans la musique qu'ils jouent sur scène, un rythme proche de celui du jazz mais avec une pointe de «Bratsch» qui apporte à la fois nostalgie et plénitude. Les artisans athéniens étant par définition des comédiens non-professionnels, leur musique scénique était entraînante, mais manquait (volontairement) de justesse et de coordination, les fausses notes, couacs et erreurs de rythmes étant prétextes à des fous rires supplémentaires de la part de cette petite troupe bien espiègle. Non pas par ce snobisme qui se développe actuellement dans le théâtre français, mais pour des raisons financières évidentes, et aussi par sympathie pour la communauté théâtrale corrézienne qui l'accueille, deux de ces artisans étaient de «vrais» acteurs amateurs. Ayant cependant une solide pratique théâtrale derrière eux, ils ont réussi à se fondre dans le groupe tout en imprimant une certaine crainte d'être maladroit ou moins performant que les autres, ce qui va dans le sens des personnages de la pièce.

Créée en juillet 1999 au Festival du Pont du Bonhomme en Bretagne, Hamlet sur la route est donc la troisième mise en scène shakespearienne de Paul Gólub. Elle a circulé dans un certain nombre de lieux, dont Le Théâtre du Soleil, et va continuer sa route par la suite. La traduction de Jean-Michel Déprats a été allégée pour correspondre aux quatre acteurs, qui, selon le principe dramaturgique, sont les seuls rescapés d'une plus grande troupe itinérante dont les autres membres se seraient dispersés suite à des menaces terribles mais non élucidées. Un prologue rapide a été introduit qui montre le désarroi des quelques acteurs restants et surtout la déception de l'actrice qui a effectué la mise en scène, Clémentine Yelnik, 
qui, parcourant les rangs de l'auditoire, explique ses intentions de mise en scène tout en s'excusant pour cette annulation. Est-ce exact ? Le spectacle aura-t-il vraiment lieu ? Est-il effectivement annulé ? Dès l'ouverture de cette pièce qui dure $2 \mathrm{~h} 30$ sans entracte, le spectateur est plongé dans le doute et l'ambiguïté. Le malaise que les acteurs sont censés ressentir replace bien dans le contexte trouble de la pièce, dans ce royaume de Danemark qui est assailli d'ennemis potentiels venant prendre leur revanche et de citoyens qui croient voir des spectres partout.

Paul GOLUB. C'était mon intention de faire un spectacle avec une distribution réduite, guidé par des raisons économiques évidentes, mais aussi artistiques. De cette façon, chaque acteur aurait à prendre en charge plusieurs rôles, ce qui pourrait mettre en valeur la théâtralité pure. Je sortais de l'aventure du Songe et de Macbeth, et après ces spectacles aux distributions importantes, je voulais réduire l'angle d'attaque. Surtout que, à certains égards, je n'étais pas complètement content de la mise en scène de Macbeth, car je ne crois pas avoir atteint le nerf secret de cette pièce, de sa forme tragique. Bien que mon approche de Hamlet peut paraître plus volontariste, par l'utilisation de seulement quatre comédiens, de marionnettes et du film, je crois que nous sommes allés plus loin dans l'interrogation de ce qu'est la tragédie shakespearienne. Finalement, on a été plus fidèle au sens profond de la pièce, mais aussi à la forme qui bascule entre l'intensité dramatique et le rire libératoire mais néanmoins tragique.

Isabelle SCHWARTZ-GASTINE. Déjà dans tes deux autres mises en scène shakespeariennes tu avais fait interpréter plusieurs rôles par les acteurs. C'est un plaisir pour le spectateur de voir la transformation de l'acteur qui peut passer d'un personnage à un autre. Une gestuelle particulière ou bien un élément de costume suffit à faire sentir la différence, l'art du comédien fait le reste. C'est le cas de Clémentine Yelnik qui interprète Gertrude, et qui n'a qu'à poser une couronne de fleurs sur sa chevelure dénouée pour être une Ophélie très crédible. De même, la même actrice passe du fossoyeur gouailleur à l'homme d'église en sortant de la trappe pleine de terre qui représente la tombe d'Ophélie. Les plaisanteries font place aux paroles sacrées, et dans ce cas précis, restent bien en mémoire.

Paul GOLUB. La pièce Hamlet et le personnage Hamlet interrogent constamment ce qu'on pourrait appeler la distinction entre vérité et illusion. C'est omniprésent dans le texte, allant du célèbre «être ou ne pas 
être» jusqu'aux métaphores théâtrales qui traversent la pièce de long en large ou de l'intérieur, comme les conseils d'Hamlet aux comédiens itinérants sur la bonne manière de jouer, etc.

Shakespeare nous fait, bien sûr, prendre conscience du fait qu'on regarde du théâtre, cette boîte à illusions qui tend son miroir fracturé vérs le monde, mais il le fait comme le personnage d'Hamlet, avec un amour et un plaisir presque enfantins, par exemple lorsqu'il bâtit la pièce à l'intérieur de la pièce. En même temps, tout en jouant avec ces procédés, Shakespeare a un message très important pour nous : il n'est peut-être pas si simple de distinguer ce qui est vrai de ce qui est faux; le théâtre, de la vie ; la vérité, du jeu. Pour le personnage d'Hamlet, ce n'est pas seulement un jeu, mais en effet une question de vie ou de mort d'essayer de distinguer qui lui ment et qui lui dit la vérité, qui porte tel ou tel masque, qui écoute derrière le fameux «arras» avec peut-être un couteau dans la main.:.

Au niveau de notre représentation, ce dédoublement joue sur plusieurs niveaux, accentuant l'interrogation déjà omniprésente dans le texte sur la soi-disant rupture entre vérité et illusion. C'est une manière très concrète de partager cela avec le public, selon un mode ludique et révélateur, de révéler la machinerie du théâtre, d'être un personnage puis soudain un autre, avec autant de crédibilité et d'ardeur. Et bien sûr, dans notre fiction à nous, celle d'une troupe réduite à quatre comédiens pour cause d'un désastre jamais divulgué, c'est une solution concrète pour résoudre les problèmes techniques du nombre de rôles à interpréter...

Isabelle SCHWARTZ-GASTINE. Il y a réduction de personnages et d'acteurs, mais recours à des subterfuges, comme les marionnettes, et ce, à plusieurs reprises.

Pour représenter Rosencrantz et Guildenstern, Clémentine Yelnik, toujours elle, garde la même blouse grise, élément de costume qu'elle utilisait déjà pour son rôle de Snout - le Mur dans Le Songe, qu'elle agrémente d'un jabot de dentelle. Elle apparaît dans l'entrebâillement du rideau de fond de scène en tenant une marionnette vêtue à l'identique au visage long et maigre qui ne laisse voir que des lèvres charnues protubérantes comme si le personnage était en train de livrer un secret ou de laisser échapper une promesse parjure. Et l'actrice parvient à calquer son visage sur la même mimique. À travers une seule et même voix, et une imitation dans la gestuelle, les deux personnages sont bel et bien présents sur scène. 
Une deuxième utilisation de marionnettes se situe tout à la fin de la pièce. Cyril Bosc, l'acteur qui interprète Horatio, passe prestement côté coulisse pour revenir par le centre en tirant un chariot : c'est l'armée des envahisseurs, formée de personnages métalliques bien alignés avec Fortimbras à leur tête, qui s'animent en bloc selon un mouvement mécanique et répétitif. Le portrait est fait de soldats indifférenciés, mais qui forment cependant un groupe compact et monolithique, prêts à lever leur arme en cadence.

Paul Golub. L'utilisation de marionnettes est une manière de changer d'échelle, de passer comme au cinéma, d'un plan à un autre, de créer ainsi des ruptures narratives qui sont comme des appels au public, de réveiller les spectateurs de leurs attentes peut-être trop classiques. En même temps, chaque marionnette á un sens précis pour nous : l'armée de Fortimbras n'est qu'une armée de pacotille, ridicule, comme un jouet pour enfant. Mais cet aspect enfantin révèle d'autant plus les dégâts réels de la guerre, comme le dit juste après Hamlet, tous ces morts pour un petit lopin de terre, quelle absurdité monstrueuse !

Isabelle SCHWARTZ-GASTINE. Il y a toujours un jeu ironique de distanciation théâtrale. On a toujours conscience qu'il s'agit d'une représentation et que ce sont des acteurs qui interprètent des personnages, et non pas une incarnation personnifiée. Un exemple : alors qu'il aborde une tirade, l'acteur qui joue Hamlet (David Ayala) s'interrompt en accusant ses camarades acteurs de faire un accompagnement musical trop soutenu qui gêne sa concentration et la réception du monologue. Quelques paroles ont été ajoutées au texte pour briser l'illusion théâtrale et ensuite pour replacer l'action dans le domaine du jeu. Et lorsque l'acteur se remet en place et reprend sa tirade depuis le début, les spectateurs prêtent une tout autre oreille au texte.

Paul GoluB. En effet, de la même manière que Shakespeare multiplie ses attaques, passant d'un mode ironique à un moment de tendresse, d'une représentation théâtrale sur scène à un monologue intériorisé, il m'a semblé important de trouver des changements de vitesse théâtraux, comme un sportif qui varie ses coups pour déstabiliser l'adversaire. Ceci répond, non pas à des effets opportunistes, mais à mon sentiment de l'extrême fébrilité et de la richesse de la pièce qui ne se contente pas d'une manière de faire ou de dire, mais qui est en mouvement continuel, et qui se renouvelle constamment, comme si le questionnement du personnage Hamlet était celui de la pièce même: «où est mon centre ?», «Qui suis-je ?». 
Isabelle SCHWARTZ-GASTINE. Le film qui va nous être montré représenté la séquence du théâtre dans le théâtre. La cour est rassemblée tournée vers le fond de scène, dos aux spectateurs, l'acteur qui joue Hamlet, de façon symbolique, se place derrière le projecteur et passe le film qui décidera de la culpabilité du roi. Ce sont les mêmes acteurs qui prennent en charge les rôles de ce film dont le contexte n'est pas celui de l'antiquité mais d'un cirque itinérant.

Paul Golub. C'est un film en $16 \mathrm{~mm}$ fait en trois jours car c'était le temps de location du matériel, en espérant avoir du beau temps. Comme éclairage, nous avions deux projecteurs de face et deux autres sur chaque côté. Il a été tourné en Corrèze près du Château de Ventadour, pas loin du site naturel utilisé pour le festival de la Luzège, dans un lieu-dit justement nommé «La Souricière».

Le dédoublement joue ici au plus fort : les personnages voient leur propre visage projeté dans ce miroir mouvant argenté qu'est notre film. Et puis, comme le film est muet, à part quelques répliques dites par les personnages qui le regardent et l'accompagnement musical que Philippe Ponty joue sur le saxo, il donne une respiration non-verbale à ce qui est finalement dans la pièce un torrent de mots et d'idées.

\section{Projection du film}

Le film est alors visionné, accompagné au saxo soprano par Philippe Ponty, directeur du Festival de la Luzège, qui interprète sa propre création musicale. David Ayala (Hamlet, dans la pièce) devient «Blanche», une version de Gertrude ; Philippe Ponty (Polonius, Laertes, le spectre et le comédien itinérant) est le clown blanc «Gonzague», une version de Hamlet père ; Cyril Bosc (Horatio, le capitaine de l'armée de Fortimbras), est «petit frère le dompteur», une version de Claudius et enfin, Clémentine Yelnik (Gertrude, Ophélie, Rosencrantz et Guildenstern, le fossoyeur, l'officiant, le metteur en scène) est «le fils».

\section{Questions après la projection}

François LAROQUE. C'était une représentation très appréciable qui donne du sens à la pièce tout en explorant le ressort théâtral du double et de la pièce dans la pièce. Ce procédé n'est pas sans évoquer des films anciens du cinéma scandinave, avec des lumières nordiques chères à Bergman ou à Dreyer, comme La nuit des forains ou bien Nosferatu.

Paul GOLUB. En effet, c'étaient nos premières références visuelles, notamment $L a$ nuit des forains. Certains personnes qui avaient vu le film 
en salle de montage se posaient réellement la question de sa provenance, et se demandaient s'il datait de l'époque du cinéma muet.

Il était très important pour nous que le film garde cette même connotation des années 20-30 qui était celle des costumes des membres de la troupe au début du spectacle. Derrière tout cela, il y avait l'idée d'un «avant-guerre», peut-être en Europe de l'Est, d'un temps d'innocence menacé par un gros désastre.

Robert ELLRODT. Dans cette version, la perception du temps est encore plus resserrée : les deux mois qui séparent le meurtre du père d'Hamlet et le mariage de sa mère deviennent littéralement deux minutes.

Paul GOLUB. Il y a déjà de cela dans Shakespeare. La pantomime jouée par les comédiens itinérants devant le roi est un raccourci théâtral nécessaire pour que le roi (et le public) reçoivent de plein fouet l'accusation de meurtre.

Peter HAPPÉ. In this film Hamlet is seen from inside, he is part of the story and unable to withdraw physically, but only mentally. He is not the complete outsider that some theatre productions make him to be.

Paul Golub. C'est vrai pour le film vu de manière isolée, hors du contexte de la pièce, mais ce n'est pas le cas lorsqu'on joue Hamlet sur la route, car notre Hamlet parle beaucoup au public, il est dans l'action mais il en sort pour commenter sa propre condition. Et puis, dans le cas de la représentation théâtrale, on voyait les quatre comédiens, notamment Hamlet, leur regard, leurs réactions par rapport au film qu'ils étaient en train de voir.

François LAROQUE. Ce procédé de démultiplier les rôles peut être considéré comme un clin d'œil aux Élisabéthains qui parfois fonctionnaient eux aussi avec des troupes réduites lorsqu'ils effectuaient des tournées.

Paul Golub. En effet, en dépit des marionnettes et du film, nous fonctionnons sur une sorte d'espace vide, un tréteau de théâtre quasimédiéval, proche dans son fonctionnement de celui du théâtre élisabéthain, globalement peu enclin aux effets et dépendant de l'art du comédien dans toute sa richesse et sa multiplicité. Au risque d'utiliser un cliché : un théâtre où le comédien est roi.

Isabelle SCHWARTZ-GASTINE. Des mises en scène qui tournent pendant plusieurs années posent le problème des interprètes. Le Songe a connu trois acteurs différents pour interpréter Bottom. Comment gérer cette donne qui est inhérente au théâtre intermittent? 
Paul Golub. C'est très difficile, surtout pour les comédiens fidèles qui restent et doivent reprendre ce qui peut paraître d'interminables répétitions; c'était le cas des artisans dans le Songe qui, notamment, avaient une partition musicale assez difficile à jouer. Heureusement, il y a le côté positif d'une énergie et d'une interprétation nouvelles qui oxygènent la troupe et révèlent la pièce autrement.

François LAROQUE. Combien avez-vous déjà donné de représentations ? Comment s'adapter à un espace différent à chaque fois que vous changez de lieu?

Paul GOLUB. On a déjà joué environ soixante fois. Les conditions sont toujours différentes. Parfois on peut intervenir plus tôt, faire des repérages et des répétitions avant la représentation, et parfois, c'est impossible. Mais on a élaboré un praticable fait spécialement pour des lieux différents, qui nous permet d'être autonomes, par exemple, les loges sont intégrées dans le décor, ce qui permet de pouvoir jouer dans une salle nue. Et les principes de mise en scène aussi se prêtent à la variété de lieux : les changements de costumes se font essentiellement à vue. Nous utilisons très peu de projecteurs.

Jean MALAPLATE. Quel usage du texte avez-vous fait?

Paul GoluB. Il s'agit donc de la traduction de Jean-Michel Déprats. Nous avons effectué beaucoup de coupes, et aussi quelques réécritures, mais pas autant que ce qui était prévu. J'avais élaboré les circonstances de départ, les raisons de la défection de la troupe, et puis, j'ai presque tout supprimé, d'abord parce que l'histoire qui liait la troupe en déroute et la performance d'Hamlet n'étaient pas assez souples, et enfin parce que je crois qu'il valait mieux laisser un doute planer sur le début. De cette façon, on percevait un malaise généralisé, une peur, qui pouvait ensuite s'appliquer à l'atmosphère de la pièce.

François LAROQUE. La traduction de Jean-Michel Déprats est un très beau texte en français qui, en même temps, reflète vraiment la version originale.

Jean-Pierre MAQUERLOT. Puisque ce film représente une action vivante et remplace une pièce de théâtre, que devient le passage dans lequel Hamlet donne des conseils aux comédiens ? Le passage sur Hécube, la tirade de Pyrrhus perdent-ils de leur pertinence?

Paul GOLUB. On a coupé les conseils d'Hamlet aux comédiens, comme on a dû couper de nombreux passages magnifiques pour une question surtout de durée, d'efficacité, d'écoute du spectacle. Par contre, on a gardé une partie importante du passage sur Hécube, surtout parce que 
le monologue d'Hamlet qui suit me semble essentiel comme baromètre de son état : Hamlet voit le comédien transporté par une émotion «factice», celle du théâtre, tandis que lui, avec des motifs réels, terribles, le meurtre de son père et le mariage de sa mère et de son oncle, ne trouve pas la force pour agir, «acter». Quelques secondes auparavant, il jouait à être fou, et là soudain, il se révèle dans toute sa douleur et toute son honnêteté.

Jean-Pierre MAQUERLOT. Quelles raisons se prêtaient à de telles manipulations ? Est-ce une adaptation? Un théâtre qui suscite l'inspiration? Est-ce que cela voudrait dire que Shakespeare génère d'autres spectacles?

Paul GOLUB. C'est la richesse des textes qui suscite la multiplicité des inventions et qui permet des réponses. Tout dépend de la position prise. C'est un point de vue négatif que de vouloir faire de la nouveauté pour la nouveauté aux dépens du sens. Mais on peut considérer qu'il y a un côté positif à entrer en dialogue avec une certaine tradition. C'est un dialogue imaginaire avec le texte, d'autres metteurs en scène, d'autres options. Et c'est aussi une façon de rendre ce texte présent et contemporain pour le public.

Gisèle VENET. Il est intéressant de constater que parmi toutes les pièces écrites à partir d'originaux, rares sont celles qui passent à la postérité et qui ont un impact. All's Well, par exemple, n'a pas été utilisée pour réécrire autre chose.

Paul GOLUB. Il est vrai que l'on trouve toujours plus d'émulation dans les grands textes. Mais souvent, ce sont les metteurs en scène qui retrouvent des textes négligés, considérés comme secondaires et qui les font revivre. On pourrait évoquer le cas de Titus, il y a de cela longtemps, lorsque Peter Brook a repris ce texte qui avait été écarté durant tant de générations.

Jean MALAPLATE. On pourrait considérer aussi qu'il y a deux genres de spectateurs.

Paul GolUB. Il est vrai que ceux qui ne connaissent pas la pièce ont tendance à s'accrocher à l'histoire, et donc ils éprouvent un soulagement parce qu'elle se révèle compréhensible et même source de plaisir. Et puis, d'autres qui, connaissant la pièce et ayant parfois vu de nombreuses mises en scène, sont à la recherche d'un nouvel aperçu, d'une nouvelle compréhension.

Margaret JONES-DAVIES. Votre mise en scène montre qu'il y a un désir de nouveauté qui a besoin d'émerger. Cherchez-vous à attirer toutes 
les catégories de spectateurs? Ou bien vous adressez-vous plus particulièrement à un public jeune?

Paul GOLUB. Nous ne visons pas particulièrement une catégorie définie de spectateurs, même si nous apprécions lorsque la pièce trouve un écho favorable parmi les spécialistes. Mais il nous arrive de jouer devant toutes sortes de public. Et là, nous avons une sorte d'idéal qui est celui du théâtre élisabéthain qui regroupait, comme il le faisait, tant de spectateurs de toutes classes, de toutes origines et, sans doute, d'âges différents. Il est important de ne pas s'enfermer dans un ghetto quel qu'il soit.

Isabelle SCHWARTZ-GASTINE. J'ai remarqué, entre autres, des représentations en province dans des lieux qui sont difficiles socialement. À Beauvais, par exemple, il s'agit d'une salle au beau milieu d'une Z.U.P. Avec un tel public, quelle est l'écoute que tu peux recevoir?

Paul GOLUB. Il est exact que dans certains cas, c'est très difficile de maintenir l'attention du public, surtout s'il s'agit en majorité de jeunes spectateurs qui ne sont pas habitués au théâtre et qui ne savent pas se concentrer dans le calme. Dans ces cas-là, on joue dans un état de crispation qui n'est pas favorable à l'acte théâtral.

Peter HAPPÉ. Shakespeare was writing for his own theatre, whereas you must find a certain adaptability to the audience through the text. In fact even in his theatre Shakespeare sometimes had to bear almost a negative environment. This is maybe why he has found such a powerful theatricality.

Paul GOLUB. Le public doit prendre une part active dans la compréhension de la pièce. À travers les nombreux monologues, les personnages se révèlent aux spectateurs. On ne peut saisir que Claudius révèle la noirceur de son âme que si on comprend que, à tel ou tel moment, le personnage joue un rôle public, porte un masque, tandis qu'à tel autre moment, il est véritablement lui-même dans la révélation de ses pensées intérieures.

Jean-Paul DEBAX. Cela dénote une très grande professionnalisation de l'écriture qui prend sa source dans une tradition plus ancienne de l'interlude joué dans les grandes maisons princières ou chez les ecclésiastiques. Je pense à Medwall, officiellement chapelain de l'archevêque de Cantorbéry, qui écrivait pour un public qu'il connaissait bien et qui savait le faire participer, dont on a retrouvé Fulgence et Lucrèce joué en 1497. Cette tradition a été utile aux dramaturges élisabéthains. 
À propos, je remarque que vous avez conservé le personnage de Fortimbras.

Jean-Pierre MAQUERLOT. On a besoin de le garder. Fortimbras évolue, il convoque les armées de rebelles. Nous ne savons pas s'il a des qualités ou des défauts, mais c'est la seule personne digne de remplacer Hamlet.

Paul Golub. Une forme de malaise plane sur la fin du texte. Fortimbras est un homme qui n'est jamais révélé. Mais c'est le génie de l'auteur et du texte de laisser une ouverture vers le futur. Le choix d'une petite marionnette habillée en kaki relève d'une option précise de notre part. Plutôt que d'être un personnage puissant, libérateur, Fortimbras est un petit chef de guérilla, agrandi seulement par sa pulsion et son envie de pouvoir. C'est une lecture qui met en question la possibilité d'un revirement positif après tant de violences et de guerres.

Gisèle VENET. Initialement, la culpabilité du vieux roi Hamlet était d'avoir éliminé le vieux Fortimbras.

Raymond GARDETTE. Le malaise vient du fait que Hamlet est roi juste à son dernier soupir.

Robert ELLRODT. Le malaise est le sentiment de gâchis exprimé par Hamlet lui-même à la fin de sa vie. C'est le retour à l'ordre banal qui conclut cette tragédie, comme celle du Roi Lear, d'ailleurs.

Paul GOLUB. Rien ne dit que Fortimbras fera un bon roi.

Raymond GARDETTE. Avez-vous gardé le spectre ?

Isabelle SCHWARTZ-GASTINE. Tu as exprimé l'idée qu'il était à présent «impossible de créer un effet crédible», que plus personne ne croyait vraiment aux revenants.

Paul GolUb. En effet, le spectre n'est plus crédible, il ne fait plus peur, comme c'était le cas, peut-être, à l'époque de Shakespeare. Et de plus, tous les moyens les plus sophistiqués ont déjà été utilisés au cinéma grâce à toutes sortes d'effets spéciaux. Mais il me semble indispensable de maintenir le spectre. Pour la première apparition, comme il s'agit d'un retour en arrière sur les répétitions de la pièce, c'est tout simplement le metteur en scène qui passe en tenant un drap sombre au-dessus de sa tête. Elle suggère un procédé qui n'est pas finalisé mais qui peut laisser trace dans l'imaginaire des spectateurs. Pour l'apparition suivante, j'ai pris à la lettre le fait qu'il apparaisse en arme, c'est Philippe Ponty qui vient sur scène, casqué, puis le visage à découvert, et là, on a gardé quasiment l'intégralité du texte du spectre. Enfin, dans l'entretien entre Gertrude et Hamlet, étant donné que le spectre n'existe que dans l'esprit d'Hamlet, je 
n'ai rien matérialisé. Ici, la présence du spectre ressemble au couteau que voit Macbeth : il est le seul à le voir, et on peut estimer que c'est une hallucination du personnage. J'ai donc choisi des solutions différentes suivant les passages, mais toutes relevant de moyens les plus sobres possibles.

Isabelle SCHWARTZ-GASTINE. Le film que l'on vient de commenter donne un aperçu de la mise en scène d'Hamlet sur la route. Il nous reste à remercier chaleureusement Paul Golub et Philippe Ponty pour leur participation à cet entretien, et de souhaiter un grand succès à la Compagnie du Volcan Bleu.

Entretien présenté par Isabelle SCHW ARTZ-GASTINE Université de Caen 